\title{
Aprendizaje de Habilidades Blandas: Percepciones de los estudiantes del Módulo Metacognitivo de la Universidad Técnica Nacional.
}

\section{Learning of Soft Skills: Students perceptions in the metacognitive module at the National Technical University}

\author{
César Toruño Arguedas* \\ *Universidad Técnica Nacional, Costa Rica. Contacto: ctoruno@utn.ac.cr \\ Referencia/ reference: \\ Toruño, C. (2020). Aprendizaje de Habilidades Blandas: Percepciones de los estudiantes del Módulo Metacognitivo de la Uni- \\ versidad Técnica Nacional. Yulök Revista de Innovación Académica. 4(1), 35-47
}

Recibido: 16 de diciembre del 2019

Aprobado: 5 de mayo del 2020

Publicado: 30 de junio del 2020

\section{Resumen}

El artículo analiza la percepción de la población estudiantil de primer ingreso de la Universidad Técnica Nacional sobre el aprendizaje de habilidades blandas en el Módulo Metacognitivo del Ciclo Introductorio 2018. La implementación de este módulo, centrado en el desarrollo de habilidades blandas, es una experiencia única en el país y en la región latinoamericana, motivo por el cual resulta relevante determinar la percepción de los estudiantes sobre la experiencia de aprendizaje y el dominio de las habilidades blandas. El estudio tuvo un enfoque mixto, un alcance exploratorio y utilizó un cuestionario digital aplicado con un muestreo por conveniencia, esto último permitió, sobre una población total de 3660 estudiantes, que se obtuviera una participación de 1532 informantes en el cuestionario de implementación y 1 014 respuestas en el seguimiento. Los resultados evidencian que los estudiantes de primer ingreso de la UTN perciben positivamente la vivencia de aprendizaje del Módulo, mejoras en su dominio de competencias blandas y relevancia en su proceso de transición a la vida universitaria, asimismo se establecen posibilidades de mejora en los aprendizajes de competencias específicas y el proceso de transición a los cursos de carrera.

Palabras clave: Educación Superior, Habilidades Blandas, Ciclo Introductorio, Módulo Metacognitivo, Transición a la Vida Universitaria, Éxito Académico.

\section{Abstract}

This article analyses the perception of first-year students of the National Technological University (UTN) on learning soft skills capacities in the Metacognitive Module of the Introductory Cycle 2018. The implementation of this module, focused in the development of soft skills, is a unique experience in the country and in the region, which is the reason why it is relevant to establish an idea of the student's perception in this field and their dominance of the matter. The study had a mix approach, an exploratory scope and used a digital survey apply with a convenience sample. Of the total of students (3360), 1532 were informant participants in the implementation of the survey with 1014 responses in the tracing. The results show that the first-year students of the UTN perceive positively the learning experience of the Module, improvements in their mastery of soft skills and relevance in their transition to university life, as well as establishing possibilities for improvement in the learning of specific competences and the process of transition to career courses.

Keywords: Higher education, Soft Skills, Introductory Cycle, Metacognitive Module, Transition to University Life, Academic success. 


\section{Introducción}

El presente artículo sistematiza los resultados obtenidos del estudio realizado por el Programa Éxito Académico sobre la percepción de la población estudiantil de primer ingreso de la Universidad Técnica Nacional (UTN) acerca del aprendizaje de habilidades blandas en el Módulo Metacognitivo del Ciclo Introductorio 2018.

El Ciclo Introductorio es una estrategia pedagógica implementada por la UTN con el objetivo de desarrollar competencias básicas requeridas por los estudiantes de primer ingreso para el éxito académico en su vida universitaria. Específicamente, el Consejo Universitario estableció en el acuerdo 8-13-2016 de la Sesión Ordinaria N. ${ }^{\circ} 13-2016$ del 29 de junio de 2016, que el propósito de este Ciclo "está centrado en que los estudiantes adquieran las competencias y conocimientos básicos necesarios para desarrollar los procesos metacognitivos, de lógica y crítica que se requieren en el proceso de formación".

El Ciclo Introductorio, implementado por primera vez en el año 2018, se compone de un Módulo Metacognitivo y un Módulo Nivelatorio en las áreas de Inglés, Matemáticas, Tecnología de la Imagen y, en el caso de la Sede Atenas, nivelatorios específicos de carreras.

El Módulo Metacognitivo, objeto del presente artículo, es una propuesta única en la educación superior costarricense al ser diseñado en conjunto por la Universidad Técnica Nacional y la Fundación Omar Dengo (instancia con experiencia en el desarrollo de educación por competencias y mediación basada en proyectos) para incorporar el desarrollo de habilidades blandas en la formación universitaria a partir de un curso específico para estudiantes de primer ingreso como primer paso en su proceso de formación académica y profesional.

El Módulo se caracteriza por una metodología de aprendizaje basada en proyectos e implementar una evaluación cualitativa, así como ser obligatorio para todos los estudiantes de primer ingreso de la universidad y una organización de competencias y estructura didáctica en los siguientes ejes: Maneras de Pensar (indagación, pensamiento crítico, argumentación científica, resolución de problemas), Maneras de Trabajar (trabajo colaborativo) y Herramientas para Trabajar (alfabetización digital).

Este artículo, adquiere relevancia académica por cuanto sistematiza las percepciones sobre el Módulo Metacog- nitivo en su primera implementación permitiendo establecer un marco de comprensión sobre su importancia, desarrollo e implicaciones desde la población estudiantil. Además, este proceso es pionero dentro de la institución al establecer una investigación durante la implementación y el seguimiento de una reforma curricular; por lo que adquiere una relevancia administrativa-pedagógica para la toma de decisiones vinculadas a las estrategias de inclusión para el éxito académico

A continuación, el artículo desarrollará una síntesis de referentes teóricos del estudio, metodología, discusión de resultados y conclusiones.

\section{Referente Teórico}

Uno de los principales desafíos de las universidades estatales, y en general de la educación superior, es la transición de la población estudiantil de la educación diversificada a la universidad, con implicaciones en los resultados del rendimiento y del éxito académico en general. $\mathrm{Al}$ respecto, un análisis de los resultados obtenidos por los estudiantes de primer ingreso de las universidades estatales coincide con estudios internacionales y concluyen lo siguiente:

La mayoría de los estudiantes que abandonan sus estudios en las universidades públicas de Costa Rica lo hacen durante los primeros ciclos lectivos. En la UCR, la UNA y el TEC el comportamiento del patrón de matrícula es similar: la curva tiene mayor pendiente entre el primero y segundo año, reflejando una salida más pronunciada al inicio de la vida universitaria de los estudiantes recién ingresados, y luego crece paulatinamente hasta convertirse en el fenómeno mayoritario a partir del quinto o sexto año de estudio. (Programa Estado de la Nación, 2017, p. 272-273)

El bajo rendimiento académico y las diversas situaciones socioeconómicas promueven tasas de deserción en el estudiantado de nuevo ingreso de "casi la tercera parte en la UCR y el TEC, una tercera parte en la UNA y el $43 \%$ en la UNED" (Programa Estado de la Nación, 2017, p. 273). El bajo rendimiento y éxito académico de los estudiantes de primer ingreso en las universidades ha sido interpretado desde una posición que infiere una causalidad determinante vinculada a la formación recibida previamente.

La anterior interpretación resulta parcial para la comprensión y atención de las realidades pedagógicas, según lo plantea Silva (2011): 
El mayor peso en la decisión de abandonar o proseguir recae sobre lo que ocurre una vez que el estudiante está adentro. Es decir, lo que ocurre "antes" del ingreso es importante, pero lo es más aquello que acontece "durante" la estadía del joven en la universidad. (p. 104)

La transición a los estudios universitarios es un momento pedagógico crucial para el aseguramiento del éxito académico y, en el caso de la UTN, la asimilación de esta premisa adquiere relevancia en el marco de su política de democratización del acceso con acciones afirmativas que promueven el ingreso de estudiantes de los quintiles I y II de la estructura socioeconómica, provenientes de cantones de bajo índice de desarrollo, en condición de trabajadores y con estudios de secundaria en colegios públicos diurnos o nocturnos. Como bien afirma Silva (2011, p. 107):

Si la transición e integración a la universidad constituye un problema para los jóvenes en general, las dificultades se acentúan entre aquellos que provienen de sectores sociales desfavorecidos, ya que acumulan una serie de desventajas socioeconómicas y culturales que obstaculizan un buen desempeño académico.

Lo anterior comprende un desafío institucional pedagógico que implica la operacionalización de las premisas del Modelo Educativo de la UTN con especial atención de la formación holística y la ecoformación como ejes estructurantes de rediseños curriculares innovadores con significativas transformaciones en la mediación y evaluación de los aprendizajes.

Por lo anterior, para atender el proceso de inclusión de estudiantes de primer ingreso a la UTN, se comparten las premisas externadas por Silva (2011, p. 111) según las cuales resulta necesario y pertinente el desarrollo de lo siguiente:

(...) dispositivos de apoyo a la integración académica y social, así como estrategias que transformen la experiencia en el aula para estimular un mayor compromiso del joven con sus estudios (...). El objetivo es diseñar programas específicos que atiendan el primer año de manera integral, y no como un cúmulo de iniciativas aisladas, y que tomen en cuenta las características y dificultades particulares de este tramo. Estos programas debieran proporcionar al nuevo estudiante un enfoque coherente y completo de la institución, sus procesos y dinámicas.

A partir de estas premisas, la estrategia de atención a la transición a la vida universitaria de la UTN (liderada por el Programa Éxito Académico) se caracteriza por el desarrollo de un Ciclo Introductorio obligatorio para estudiantes de primer ingreso de todas las carreras de la universidad en el cual se desarrollan las habilidades blandas (Módulo Metacognitivo) y conocimientos básicos requeridos en los cursos de diplomado o bachillerato (Módulos Nivelatorios).

En el caso de las habilidades blandas, desde el inicio del siglo XXI existen propuestas para la educación superior latinoamericana siendo referente el Proyecto Tuning (2007) en el cual se desarrollaron competencias genéricas y disciplinares para diversas carreras universitarias con el objetivo de establecer una estrategia latinoamericana de formación de competencias reconocidas por diversas instituciones universitarias. Entre las competencias genéricas, el Proyecto Tunning (2007), reconoce la capacidad de abstracción, análisis y síntesis, capacidad de comunicación oral y escrita, habilidades en el uso de las tecnologías de la información, capacidad de investigación, habilidades para buscar, procesar y analizar información, capacidad para actuar en nuevas situaciones, capacidad creativa, capacidad para resolver problemas, capacidad para tomar decisiones y capacidad de trabajo en equipo. La incursión del Proyecto Tuning es un reflejo de las nuevas realidades y demandas socioproductivas de los países en el marco de los procesos de globalización y revolución tecnológica (con sus acelerados cambios en las funciones de los trabajadores). Estas realidades emergentes exigen cambios en los procesos de formación profesional universitaria, siendo fundamental la incorporación de las habilidades blandas como requisito de pertinencia en el mercado productivo (Errázuriz, 2018) y al mismo tiempo es un requisito de formación académica para la mejora de los procesos de aprendizaje en las universidades comprometidas con nuevas formas epistémicas, como el caso del modelo educativo de la UTN (Universidad Técnica Nacional, 2016).

El requerimiento de la formación en habilidades blandas implica, para las universidades, la consideración de una formación desde la inclusión de estrategias de aprendizaje, capacidad de generación del conocimiento (pensamiento crítico, habilidad profesional), estrategias para desarrollar habilidad (multidisciplinario e interdisciplinario, exposición a nuevos ambientes y perspectivas, aceptar riesgos), creatividad, pensamiento sistemático y liderazgo (Garza, 2018). Esto es posible mediante el rediseño de planes de estudio, incorporación de ejes transversales en las mallas curriculares, constitución de cursos 
específicos, modificación de la mediación y evaluación de los cursos (siendo esta la más recomendable para el desarrollo continúo de las habilidades), talleres de formación complementaria, actividades extracurriculares y contextualización curricular, comprendida como "la transposición del diseño curricular a la realidad del aula, mediado por la planificación del docente (planeamiento didáctico o de contextualización curricular) y la acción pasiva-activa del estudiante" (Toruño, 2020, p. 55)

En el ámbito universitario existen propuestas para el desarrollo de habilidades blandas en asignaturas introductorias (González, Ferreira y Barranco, 2018) o la importancia del Aprendizaje Basado en Problemas en las áreas de ingenierías (López, Mota y Alvarado, 2018), sin embargo el caso de la propuesta del Módulo Metacognitivo de la UTN resulta único en la región latinoamericana por cuanto: a) es un curso obligatorio para estudiantes de primer ingreso, b) su objetivo es el desarrollo de habilidades blandas, c) no posee evaluación cuantitativa y, d) cuenta con una estructura pedagógica y metodológica diseñada por un par externo con experiencia en la capacitación de habilidades blandas.

Específicamente, el Módulo Metacognitivo es producto de un diseño curricular elaborado por la Fundación Omar Dengo para la Universidad Técnica Nacional a partir de un diagnóstico de necesidades de formación de habilidades blandas para estudiantes. Es importante indicar que la denominación de módulo es justificada por la integración en la formulación epistémica, metodológica y evaluativa de Metacognitivo y Nivelatorios.

El módulo es de curso obligatorio para toda la población estudiantil de primer ingreso, se compone de 13 sesiones presenciales (una por semana) con una duración de cuatro horas presenciales por sesión, es impartido por profesores de las diferentes carreras que son capacitados para ser facilitadores del proceso de aprendizaje del módulo. Para alcanzar una homologación didáctica, se diseñaron las Guías (fichas) Didácticas de cada sesión (incluye el paso a paso de cada actividad, distribución del tiempo y estructura de evaluación), Guías de trabajo independiente para el estudiante, instrumentos de evaluación, los materiales didácticos de cada una de las sesiones y material de apoyo complementario para docentes y estudiantes.

La estructura curricular del módulo desarrolla las habilidades de Maneras de Pensar (indagación, pensamiento crítico, argumentación científica y resolución de proble- mas), Maneras de Trabajar (trabajo en equipo) y Herramientas para Trabajar (alfabetización digital) mediante una estrategia de pensamiento sistemático compuesto por la definición de objetivos-problemas-propósitos, elaboración de un plan de acción, ejecución de un plan de acción de forma controlada y monitoreada (autorregulación) y evaluación o reflexión sobre el proceso.

El pensamiento sistemático y las habilidades se implementan durante las 13 sesiones del módulo a partir de una mediación sustentada en el Aprendizaje Basado en Problemas para el desarrollo de dos Proyectos (mi trabajo soñado y el uso de redes sociales) y una evaluación cualitativa centrada de brindar una realimentación permanente al estudiante. Sobre este último elemento, se debe destacar que el Módulo Metacognitivo y los Módulos Nivelatorios implementan una evaluación cualitativa sin ponderación cuantitativa, siendo la primera experiencia de la educación superior costarricense en integrar un ciclo completo de módulos o cursos de un plan de estudio con evaluación cualitativa.

\section{Metodología}

La investigación realizada en el marco de la implementación y seguimiento del Módulo Metacognitivo de la UTN del año 2018 se caracterizó por utilizar el enfoque mixto con un alcance exploratorio en el tanto permitió "obtener información para realizar una investigación más completa de un contexto particular, investigar nuevos problemas, identificar conceptos o variables promisorias, establecer prioridades para investigaciones futuras" (Hernández, Fernández y Baptista, 2010). Sin embargo, para el presente artículo se presentan los resultados cuantitativos del estudio.

Para la recolección de información, sobre una población total de 3660 estudiantes, se utilizaron dos cuestionarios digitales aplicados con un muestreo por conveniencia por cuanto "selecciona participantes por uno o varios propósitos y no pretende que los casos sean representativos de la población" (Hernández, Fernández y Baptista, 2010, p. 171). En ambos casos, los cuestionarios fueron respondidos de forma voluntaria y el encabezado indicaba las características del consentimiento informado.

El primer cuestionario aplicado se realizó en la cuarta semana de abril de 2018 (cierre del cuatrimestre) para sistematizar la percepción de los estudiantes durante la implementación del Módulo Metacognitivo. En este 
cuestionario se consultó la percepción de los estudiantes sobre el dominio de habilidades, valoraciones sobre la contextualización curricular y generales sobre el módulo.

El cuestionario se integra por tres subapartados. El primero, compuesto de siete preguntas, indagaba por información general; el segundo subapartado contenía 17 preguntas para determinar la percepción de los estudiantes sobre dominio de habilidades blandas (antes y después del Módulo), condiciones de contextualización curricular, características didácticas y elementos de valoración general de labor docente e importancia del módulo; el último subapartado contenía dos preguntas generales para valorar percepciones generales desde una posición cualitativa.

Sobre las preguntas vinculadas con la percepción de dominio de habilidades, es importante destacar que ante cada una de las habilidades se les consultó a los estudiantes sobre el grado de dominio que tenía previamente a iniciar el Módulo y que grado de dominio tenía al finalizar el Módulo (momento de implementación del cuestionario). Finalmente, se debe indicar que 1532 estudiantes respondieron este cuestionario, en este caso hubo una distribución por género de 896 mujeres $(58,5 \%$ ) y 636 hombres $(41,5 \%)$ y una distribución por sede universitaria de la siguiente manera: Sede Central 610 (39, $8 \%$ ), Sede Pacífico 349 (22,8 \%), Sede Guanacaste 223 (14,6 \%), Sede San Carlos 222 (14,5\%) y Sede Atenas 128 (8,4 \%). Después de un análisis general, se eliminaron 14 formularios por estar incompletos.

El segundo cuestionario se aplicó en la cuarta semana de agosto de 2018 (cierre del segundo cuatrimestre) dirigido a establecer la percepción de los estudiantes sobre la utilidad del Módulo Metacognitivo en los cursos del segundo cuatrimestre, motivo por el cual se consultó sobre la utilidad del Módulo y el dominio de habilidades y destrezas vinculadas con habilidades blandas. El cuestionario se integró de tres subapartados, en el primero de ellos se establecieron cinco preguntas de información general; el segundo subapartado se integró de cuatro preguntas de valoración general sobre la importancia del Módulo Metacognitivo en función de habilidades y destrezas para los cursos del segundo cuatrimestre y, finalmente, un bloque de dos preguntas cualitativas para valoraciones generales que permitieran una evaluación cualitativa.

El segundo cuestionario fue respondido por 1014 estudiantes con una distribución de $612(60,4 \%)$ mujeres y
$402(36,6 \%)$ hombres. Con respecto a la sede en la que cursa estudios, $410(40,4 \%)$ eran estudiantes de la Sede Central, 244 (24,1 \%) Sede San Carlos, 149 (14,7\%) Sede Guanacaste, 145 (14,3\%) Sede Pacífico y 66 (6,5 $\%)$ Sede Atenas. Los datos obtenidos en los dos fueron analizados mediante la interpretación de cuadros estadísticos y gráficos

\section{Resultados y discusión}

El estudio integra resultados durante la implementación (primer cuatrimestre) y el seguimiento (segundo cuatrimestre) de la población estudiantil que cursó el Módulo Metacognitivo durante en el año 2018. A continuación, se abordarán los principales resultados obtenidos en cada etapa de estudio.

\section{Resultados en la Implementación}

En el estudio de implementación, el análisis de los datos obtenidos de 1532 respuestas estableció la necesidad de suprimir 14 formularios por encontrarse incompletos, motivo por el cual el presente análisis se realiza con 1518 respuestas $(41,5 \%$ de la población total de 3660 estudiantes de primer ingreso) para establecer la percepción de los estudiantes sobre la vivencia del aprendizaje y el dominio de las competencias blandas.

La vivencia del aprendizaje fue analizada desde la coherencia de la contextualización curricular con respecto al diseño del Módulo y el desarrollo de habilidades y destrezas. Para tal fin, se consultó sobre la importancia que han tenido la práctica y realimentación, trabajos extraclases, presentación de proyectos y materiales, además de la reflexión sobre la propia práctica en el marco de la vivencia del aprendizaje.

La importancia de práctica y realimentación como parte de las actividades de aprendizaje es valorada como baja y muy baja por el $8,9 \%$ de los estudiantes, en forma regular por un $22,8 \%$ y positivamente (alta y muy alta) por un $68,8 \%$; por su parte, la presentación de proyectos y materiales obtuvo una valoración negativa del 9,3\%, un $22,2 \%$ la percibe de forma regular y el $68,5 \%$ percibe una importancia alta y muy alta durante sus procesos de aprendizaje, esta última cifra se mantiene al ser consultados sobre la importancia del Trabajo Extra Clase. En general, se debe considerar que el 68,5\% de la población estudiantil califica como alta y muy alta la importancia de estas actividades durante su proceso de aprendizaje 
permite establecer una ejecución coherente con respecto a la metodología del Módulo, principalmente con la priorización de Aprendizaje Basado en Proyectos.

Sumado a lo anterior, se les consultó a los estudiantes sobre la importancia que ha tenido la reflexión sobre la propia práctica para la construcción de sus aprendizajes ante lo cual el 2,9\% de los estudiantes percibieron una importancia muy baja, el 6,8 \% baja, el $23 \%$ media, el $36,7 \%$ alta y el $30,6 \%$ muy alta. Al ser un objetivo tácito de la metacognición lograr la conciencia y reflexión de los procesos que realizaba cada persona para su aprendizaje se destaca que 9,7\% de los estudiantes asignen una baja importancia a esta acción durante sus procesos aprendizaje.

El hecho de que el $67,3 \%$ de la población estudiantil reconozca con una importancia alta y muy alta, la reflexión sobre la práctica evidencia el cumplimiento de la propuesta metacognitiva mediante las actividades didácticas implementadas en las sesiones.

En consideración de que la estrategia metodológica del Módulo Metacognitivo establece como eje estructurante el trabajo colaborativo, se consultó a los estudiantes sobre su percepción del ambiente del grupo durante las diferentes sesiones, esto con el objetivo de visibilizar las características de socialización. Para tal fin se les solicitó indicar su acuerdo (de acuerdo y totalmente de acuerdo) o desacuerdo (en desacuerdo y totalmente desacuerdo) con diferentes afirmaciones, a continuación, se analizarán los resultados a partir de la sumatoria de afirmaciones positivas.

Ante la afirmación, "todos han participado con interés y motivación" el 68,8\% de los informantes se manifestó de acuerdo o muy de acuerdo y sobre la frase "sabíamos en todo momento lo que se esperaba de nosotros" se presentó de acuerdo o muy de acuerdo con el 66,5\%. El aval de ambas premisas permite analizar las posibilidades de aprendizaje a partir del interés y motivación, así como a la claridad de la estructura didáctica reconocida por los estudiantes.

Sobre la afirmación "ha habido un ambiente de cooperación en las diferentes actividades de aprendizaje" el $85,7 \%$ de los estudiantes manifestaron estar de acuerdo o muy de acuerdo. Esto se ratifica al contrastar los datos obtenidos al consultar a los estudiantes sobre la afirmación "las tensiones y conflictos se han resuelto favorable- mente" lo cual es avalado por el $87 \%$ de los estudiantes.

Lo anterior adquiere relevancia por la posible vinculación del trabajo en grupo, el aprendizaje colaborativo y el aprendizaje basado en proyectos que sustentan el Módulo, lo cual pudo favorecer la construcción de espacios de aprendizaje colectivo por una socialización positiva tanto para enfrentar el aprendizaje, como diferencias a lo interno de los grupos de trabajo o de la clase.

Ante la afirmación de que "se han percibido las actividades de aprendizaje como provechosas" el 78,5\% de los estudiantes manifestaron estar de acuerdo o muy de acuerdo. Asimismo, sobre la afirmación de que se han "desarrollado actividades innovadoras" el $80,3 \%$ de los estudiantes manifestaron estar de acuerdo o muy de acuerdo.

La comprensión por parte de la población estudiantil de lo provechoso de cada actividad de aprendizaje y su innovación no solo favorece el aprendizaje significativo, sino que permite ratificar la relevancia y pertinencia de la estructura metodológica del módulo para estudiantes de primer ingreso.

Finalmente, sobre la contextualización curricular, se consultó a los estudiantes sobre su valoración del profesor o de la profesora. En este apartado un 86,3\% de los estudiantes conceden una valoración buena o muy buena a sus docentes, siendo una ponderación muy alta que se enmarca en las altas valoraciones de las actividades didácticas, el trabajo colaborativo y la innovación analizadas anteriormente.

Junto a los elementos de la contextualización curricular, se consultó a los estudiantes sobre el dominio de habilidades blandas antes y al finalizar el Módulo Metacognitivo, específicamente sobre el dominio de trabajo en equipo, identificar y resolver problemas, uso de buscadores y bases de datos, adaptación y toma de decisiones en situaciones nuevas, argumentar y debatir y, finalmente, hablar en público. El análisis se realizó desde la percepción de dominio previo y dominio al finalizar el Módulo Metacognitivo en categorías de Muy Bajo, Bajo, Medio, Alto y Muy Alto, se obtienen los resultados indicados en la tabla 1. 
Tabla 1. Percepción de la población estudiantil del Módulo Metacognitivo sobre el dominio de habilidades blandas.

\begin{tabular}{|c|c|c|c|c|c|c|c|}
\hline $\begin{array}{r}\text { Habilidad } \\
\text { blanda }\end{array}$ & $\begin{array}{l}\text { Percepción } \\
\text { de dominio }\end{array}$ & Muy Bajo & Bajo & Medio & Alto & $\begin{array}{r}\text { Muy } \\
\text { alto }\end{array}$ & $\begin{array}{r}\text { Sumatoria Alto } \\
\text { y Muy Alto }\end{array}$ \\
\hline \multirow{2}{*}{$\begin{array}{r}\text { TRABAJO } \\
\text { EN EQUIPO }\end{array}$} & $\begin{array}{r}\text { Antes de } \\
\text { iniciar el } \\
\text { módulo }\end{array}$ & 2,5 & 11,4 & 41,0 & 32,3 & 12,8 & 45,1 \\
\hline & $\begin{array}{r}\text { Al finalizar } \\
\text { el módulo }\end{array}$ & 1,1 & 1,4 & 13,6 & 51,3 & 32,6 & 83,9 \\
\hline \multirow{2}{*}{$\begin{array}{r}\text { IDENTI- } \\
\text { FICAR Y } \\
\text { RESOLVER } \\
\text { PROBLEMAS }\end{array}$} & $\begin{array}{r}\text { Antes de } \\
\text { iniciar el } \\
\text { módulo }\end{array}$ & 5,9 & 24,0 & 42,2 & 21,0 & 6,9 & 27,9 \\
\hline & $\begin{array}{r}\text { Al finalizar } \\
\text { el módulo }\end{array}$ & 0,9 & 2,0 & 22,3 & 56,9 & 18,0 & 74,9 \\
\hline \multirow{2}{*}{$\begin{array}{r}\text { USO DE BUS- } \\
\text { CADORES } \\
\text { Y BASES } \\
\text { DE DATOS }\end{array}$} & $\begin{array}{c}\text { Antes de } \\
\text { iniciar el } \\
\text { módulo }\end{array}$ & 14,2 & 25,5 & 33,2 & 18,6 & 8,4 & 27 \\
\hline & $\begin{array}{r}\text { Al finalizar } \\
\text { el módulo }\end{array}$ & 1,2 & 3,6 & 28,9 & 48,1 & 18,2 & 66,3 \\
\hline \multirow{2}{*}{$\begin{array}{r}\text { ADAPTARSE } \\
\text { A SITUACIO- } \\
\text { NES NUEVAS } \\
\text { Y TOMAR } \\
\text { DECISIONES }\end{array}$} & $\begin{array}{r}\text { Antes de } \\
\text { iniciar el } \\
\text { módulo }\end{array}$ & 4,0 & 17,1 & 44,3 & 24,6 & 9,9 & 35,5 \\
\hline & $\begin{array}{r}\text { Al finalizar } \\
\text { el módulo }\end{array}$ & $\mathbf{0 , 5}$ & 1,3 & 19,6 & 54,9 & 23,6 & 78,5 \\
\hline \multirow{2}{*}{$\begin{array}{r}\text { ARGU- } \\
\text { MENTAR Y } \\
\text { DEBATIR }\end{array}$} & $\begin{array}{r}\text { Antes de } \\
\text { iniciar el } \\
\text { módulo }\end{array}$ & 14,0 & 26,8 & 32,7 & 18,1 & 8,4 & 26,5 \\
\hline & $\begin{array}{r}\text { Al finalizar } \\
\text { el módulo }\end{array}$ & 1,0 & 2,5 & 28,8 & 48,5 & 19,2 & 67,7 \\
\hline \multirow{2}{*}{$\begin{array}{r}\text { HABLAR EN } \\
\text { PÚBLICO }\end{array}$} & $\begin{array}{r}\text { Antes de } \\
\text { iniciar el } \\
\text { módulo }\end{array}$ & 17,0 & 21,5 & 30,6 & 19,6 & 11,3 & 30,9 \\
\hline & $\begin{array}{r}\text { Al finalizar } \\
\text { el módulo }\end{array}$ & 1,1 & 4,7 & 31,2 & 42,0 & 21,0 & 63 \\
\hline
\end{tabular}

Fuente: elaboración propia, 2019.

En la competencia de trabajo en equipo, antes de iniciar el Módulo Metacognitivo, el 2,5 \% la población estudiantil percibía un dominio muy bajo, el $11,4 \%$ bajo, el $41 \%$ medio, el $32,3 \%$ alto y el $12,8 \%$ un dominio muy alto; mientras que, al finalizar el Módulo, el 1,1 \% percibía un dominio muy bajo, el 1,4\% un dominio bajo, el 13,6 \% medio, el $51,3 \%$ alto y el $32,6 \%$ muy alto.

Lo anterior evidencia que una disminución significativa de la percepción de dominio bajo (10 \% menos) y medio
$(18,7 \%$ menos) mientras que el dominio alto y muy alto pasan de representar el $45,1 \%$ al $83,9 \%$, posiblemente, vinculado a las actividades didácticas con juegos de roles en los que se indica los papeles que deben realizar cada integrante del grupo, la distribución de responsabilidades e instrumentos para realimentar a sus compañeros. La comprensión reflexiva del trabajo en grupo o trabajo colaborativo, podría ser un factor de mejora para su dinámica en los procesos de aprendizaje colectivo de los cursos de la carrera. 
Con respecto a la habilidad Identificar y Resolver Problemas, el 5,9\% de los estudiantes indicaron que antes de iniciar el Módulo tenían un dominio muy bajo, el $24 \%$ bajo, $42,2 \%$ medio, $21 \%$ alto y $6,9 \%$ muy alto; en tanto, al finalizar el Módulo, el 0,9 \% indicó un dominio muy bajo, el $2 \%$ bajo, el $22,3 \%$ medio, el $56,9 \%$ alto y el 18 $\%$ muy alto. En esta competencia, la influencia positiva del módulo se evidencia en un aumento significativo de las percepciones altas y muy altas, las cuales pasan de representar el $27,9 \%$ al $74,9 \%$, esto asociado a una mayor concientización sobre las etapas de la resolución de problemas y la implementación de ejercicios durante la experiencia de aprendizaje.

La significativa mejora de la percepción de dominio en la Identificación y Resolución de problemas evidencia la integración y aplicación de la estrategia de pensamiento sistemático (definición de objetivos-problemas-propósitos, elaboración de un plan de acción, ejecución de un plan de acción de forma controlada y monitoreada y evaluación o reflexión sobre el proceso), la cual es una estrategia implementada en las diversas sesiones del módulo y en los procesos de formulación de proyectos, todo esto impulsado desde las guías didácticas para el profesor.

Por la dinámica de aprendizaje en las diferentes sesiones, las habilidades de Trabajo Colaborativo e Identificar y Resolver Problemas se encuentran vinculadas en las actividades $y$, por tanto, se puede presumir que su dominio muestra una correlación. Al respecto, se destaca que el dominio bajo o muy bajo en ambas competencias es percibido, al finalizar el módulo, por menos del $3 \%$ de la población estudiantil y más del $74 \%$ percibe un dominio alto o muy alto.

En las habilidades tecnológicas vinculadas con el Uso de Buscadores y Bases de Datos, el 14,2 \% de la población estudiantil consideró que antes de iniciar el Módulo tenía un dominio muy bajo, el 25,5\% bajo, el 33,2 \% medio, el $18,6 \%$ alto y el $8,4 \%$ muy alto; al finalizar el módulo indican una mejora en el dominio con el 1,2 \% muy bajo, el 3,6 \% bajo, el $28,9 \%$ medio, el $48,1 \%$ alto y el 18,2 $\%$ muy alto.

En la competencia tecnológica, al igual que las anteriores, los resultados son positivos y evidencian un crecimiento de los niveles alto y muy alto inferior al promedio y una leve disminución del rango "medio", esto amerita un análisis cualitativo para determinar si se requiere in- crementar las actividades con uso tecnológico en el aula para facilitar el dominio de la competencia.

Al establecer el dominio de la competencia Argumentar y Debatir previo al Módulo Metacognitivo, el $14 \%$ de la población estudiantil percibía un dominio muy bajo, el $26,8 \%$ bajo, el $32,7 \%$ medio, el $18,1 \%$ alto y el $8,4 \%$ muy alto; al finalizar el Módulo el $1 \%$ percibía un dominio muy bajo, el 2,5\% bajo, el 28,8 \% medio, el 48,5\% alto y el 19,2\% muy alto. Los datos muestran una mejora significativa de la percepción de dominio que pasa del $26,5 \%$ alto y muy alto al $67,7 \%$ en ambas categorías al finalizar el Módulo, esto puede ser vinculado con las actividades de aprendizaje caracterizadas por proyectos, resolución de problemas, investigación y juego de roles.

Correlacionado con la competencia de Argumentación y el Debate, se analizó la percepción de los estudiantes sobre el dominio de destrezas y habilidades para hablar en público, al respecto el $17 \%$ indicó que, previo al Módulo, tenían un dominio muy bajo, el 21,5\% bajo, el 30,6 \% medio, el 19,6\% alto y el 11,3\% muy alto; al finalizar el Módulo el 1,1 \% de la población estudiantil percibía un dominio muy bajo, el 4,7\% bajo, el 31,2\% medio, el 42 $\%$ alto y el $21 \%$ muy alto.

En este apartado destaca el empoderamiento que pudo desarrollar el $33 \%$ de estudiantes que reportaban un dominio previo muy bajo o bajo y que al finalizar el Módulo solo 5,8 \% un nivel de dominio en esta escala. Esto es posible en consideración de que el juego de roles definido para todas las sesiones del módulo exige la constitución de grupos de trabajo y la distribución semanal de roles (que deben variar cada semana, es decir, ningún estudiante puede repetir el rol en las semanas siguientes) como relator; siendo este último el encargado de hablar en público, defender posiciones y explicar la dinámica-productos del grupo.

Las habilidades de Argumentar y Debatir con las Habilidades para Hablar en Público pueden ser analizadas con correlaciones por las dinámicas de las actividades de aprendizaje en el aula, y explicitadas en el Programa del Módulo y las guías didácticas del cuerpo docente, ligados con la discusión y presentación de proyectos, investigaciones y la resolución colaborativa de problemas. En este contexto, destaca que una media de $45 \%$ de la población estudiantil reportaban un dominio muy bajo o bajo en ambas competencias previo al Módulo y al finalizar 
una media de $1 \%$ percibió un dominio muy bajo y solamente el 3,6\% un dominio bajo, sumado a percepciones de dominio alto y muy alto superiores al $60 \%$ en ambas categorías.

Finalmente, la población estudiantil fue consultada sobre el dominio de la competencia Adaptación a Situaciones Nuevas y Toma de Decisiones. En este apartado, previo al Módulo, el $4 \%$ de los estudiantes percibían un dominio muy bajo, el 17,1 \% bajo, el 44,3 \% medio, el 24,6\% alto y el 9,9\% muy alto; al finalizar el Módulo, el 0,5\% percibieron un dominio muy bajo, el 1,3\% bajo, el 19,6 $\%$ medio, el $54,9 \%$ alto y el $23,6 \%$ muy alto.

Alcanzar una percepción de dominio alta o muy alta del $78,5 \%$ de los estudiantes en la competencia de Adaptación y Toma de Decisiones evidencia un impacto del Módulo en la transición a la vida universitaria por parte de estudiantes de primer ingreso que debe ser profundizado en una investigación cualitativa, principalmente en su posible vinculación con la creación de mecanismos de socialización entre pares, acumulación de capital cultural para enfrentar los cursos iniciales de las carreras y la construcción de una identidad universitaria (individual y colectiva) motivada, no exclusivamente, por la propuesta metodológica y epistémica del módulo así como la vivencia de las experiencias socioemocionales vinculadas al proceso de aprendizaje desarrollado durante el cuatrimestre.

En síntesis, el instrumento aplicado durante la implementación de la primera experiencia del Módulo Metacognitivo (2018), evidencia que la contextualización curricular (práctica, realimentación y reflexión) percibida por 7 de cada 10 estudiantes es coherente con la propuesta epistémica y metodológica del programa, 8 de cada 10 estudiantes percibe el trabajo colaborativo (elemento fundamental del Aprendizaje Basado en Proyectos) y la innovación de las actividades. Con respecto al dominio de habilidades blandas, la percepción de mejora (en las percepciones categorizadas como Alto y Muy Alto) entre al antes y el después es entre dos y tres veces mayor al finalizar el Módulo,

\section{Resultados seguimiento}

En el apartado de seguimiento, al finalizar el segundo cuatrimestre (4 meses después de concluir el Módulo Metacognitivo), se recolectó la información 1014 informantes, pero 11 formularios fueron suprimidos por encontrarse incompletos, motivo por el cual se analizó la información de 1003 estudiantes $(27,4 \%$ del total de estudiantes que cursaron el Módulo) para establecer percepción sobre la vinculación e influencia (baja, media o alta) del Módulo Metacognitivo con los cursos de la carrera; específicamente, en las áreas de facilitar la transición a la universidad, socialización, formas de aprender y estudiar y utilidad para los cursos del segundo cuatrimestre.

A continuación, se expondrá el análisis de resultados sobre la consulta de tres premisas básicas de vinculación (facilitar mi transición a la universidad, mejorar mis formas de aprender, relación con mis compañeros) en las cuales se consultó a partir de los indicadores de baja, media y alta. Para el análisis, se ha sumado el resultado de las categorías medio y alto en consideración de que, al correlacionarse con las respuestas obtenidas en las preguntas específicas para determinar la vinculación, se obtiene un porcentaje equivalente, evidenciando una tendencia en la percepción.

La utilidad del Módulo Metacognitivo para "facilitar mi transición a la universidad" es reconocida positivamente por el $76,9 \%$ de la población estudiantil (41,1 \% determinaron una influencia media y el $35,8 \%$ alta) y el 23,1 $\%$ reconocen una influencia baja. A partir de estos datos, se puede inferir el impacto del Módulo para facilitar transición a la vida universitaria a partir del desarrollo de habilidades blandas que influyen en elementos como empoderamiento, confianza, socialización, disciplina de estudio, aprendizaje colaborativo y construcción de identidades colectivas (grupo, sede y universidad); el análisis de la correlación de estas variables (Módulo y transición a la vida universitaria) requiere un estudio cualitativo con seguimiento de casos

Asimismo, facilitar la transición a la universidad se vincula con procesos académicos y sociales. En este apartado, sobre la utilidad del Módulo para "Mejorar mis formas de aprender y estudiar" el 72,5\% de los informantes reconoce una utilidad media y alta (el 40,4\% media y el $32,1 \%$ alta) y el $27,5 \%$ baja; datos que permiten inferir una posible correlación entre la transición a la vida universitaria, el Módulo y la mejora de las técnicas de estudio y la disciplina de estudio.

La importancia del Módulo y del Ciclo Introductorio en general, para la mejora de las formas de aprender y estudiar es motivado durante el Módulo Metacognitivo a partir de la implementación de una evaluación cualitati- 
La influencia del Módulo en los cursos del segundo cuatrimestre del 2018 se analizó a partir del establecimiento del grado de acuerdo (Totalmente en Desacuerdo, En Desacuerdo, De Acuerdo y Totalmente de Acuerdo) de la población estudiantil sobre el desarrollo de habilidades, destrezas y competencias generales del Módulo. A continuación, y con el objetivo de sintetizar los resultados, se expondrán en la tabla 2, a partir de la sumatoria de categorías positivas (De Acuerdo y Totalmente de Acuerdo).

Tal cual puede apreciarse en la tabla 2, en el área de Habilidades y Destrezas para la comunicación en los procesos de aprendizajes, el 60,8\% de los estudiantes responden positivamente a la afirmación "Mejoró mi capacidad para hablar en público", el 61,8 \% afirma que "Influyó en mi confianza y capacidad para argumentar y debatir en clase" y ante la afirmación "Me permitió desarrollar mejores presentaciones" el 63,3 \% afirmó estar de acuerdo o muy de acuerdo.

Tabla 2. Percepción de la población estudiantil sobre la influencia del Módulo Metacognitivo en la dinámica de aprendizaje de los cursos del segundo cuatrimestre.

\begin{tabular}{|l|c|}
\hline $\begin{array}{l}\text { Influencia del Módulo Metacognitivo en los procesos de aprendiza- } \\
\text { je de cursos del segundo cuatrimestre (mayo-agosto) del 2018 }\end{array}$ & $\begin{array}{c}\text { Porcentaje de estu- } \\
\text { diantes que respondie- } \\
\text { ron De Acuerdo y To- } \\
\text { talmente de Acuerdo }\end{array}$ \\
\hline Me permitió desarrollar mejores presentaciones en los cursos & 63,3 \\
\hline Me brindó herramientas para realizar mejores investigaciones en los cursos & 61,8 \\
\hline $\begin{array}{l}\text { Favoreció mi capacidad para usar buscadores especializados (bases de datos, Google } \\
\text { Académico u otro) }\end{array}$ & 59,9 \\
\hline Influyó en mi confianza y capacidad para argumentar y debatir en clase & 61,8 \\
\hline Mejoró la forma en cómo participo en el trabajo en equipo & 65,2 \\
\hline Mejoró mi capacidad de identificar problemas y establecer rutas para crear soluciones & 59,6 \\
\hline $\begin{array}{l}\text { Me permitió mejorar mi capacidad para adaptarme a las nuevas situaciones y exigencias } \\
\text { de los cursos }\end{array}$ & 60,0 \\
\hline Mejoró mi capacidad para hablar en público & 60,8 \\
\hline
\end{tabular}

Fuente: elaboración propia, 2019. 
El hecho de que el $60 \%$ de los estudiantes reconozcan la influencia de las habilidades y destrezas para la comunicación en los procesos de aprendizaje (hablar en público, defender ideas y exponer) evidencia la potencialidad del Módulo para desarrollar un empoderamiento de los estudiantes en este apartado. El desarrollo de esta habilidad facilita las posibilidades de vivencias de aprendizaje crítico, así como la implementación de metodologías coherentes con el Modelo Educativo de la UTN.

Especial atención requiere la mejora de la habilidad para hablar en público, en el tanto significa una ruptura cualitativa en los apartados psicológicos y sociales de la vivencia de los aprendizajes en aula. El valor intangible del empoderamiento del estudiante para exponer ante sus pares (con un valor agregado de este proceso desde el frente de la clase), es un proceso vital para la vida académica, profesional y personal; altamente relevante en consideración de una población estudiantil donde más del $90 \%$ proviene de la educación pública y poco más del $50 \%$ es primera generación universitaria en sus hogares.

En relación con las habilidades-destrezas para la investigación, el 59,6 \% de los estudiantes manifestaron estar de acuerdo o totalmente de acuerdo con la afirmación "Mejoró mi capacidad de identificar problemas y establecer rutas para crear soluciones". Esto se vincula con el desarrollo de estructuras cognitivas complejas para el análisis y evaluación de situaciones, evidenciando un proceso de reconocimiento de fases y acciones para la resolución de problemas.

Ante la afirmación "Me brindó herramientas para realizar mejores investigaciones en los cursos" el 61,8 \% de los estudiantes manifestaron estar de acuerdo o totalmente de acuerdo y ante la premisa "Favoreció mi capacidad para usar buscadores especializados" las respuestas positivas corresponden al 59,9\% de estudiantes.

Los datos permiten establecer una percepción de mejora, por parte de los estudiantes, en su capacidad de investigación, uso de buscadores y resolución de problemas. De esta forma, se cumple la estructura metodológica y fin pedagógico del Módulo para que los estudiantes puedan establecer un problema, crear una estrategia de solución, utilizar bases de datos para obtener información relevante para la atención del problema y la sistematización de la información mediante las etapas de investigación básica en un nivel universitario.
Las áreas de proceso de comunicación e investigación encuentran como ejes estructurales las habilidades de trabajo en equipo y adaptación a nuevas situaciones. En la primera de estas, ante la afirmación "Mejoró la forma en cómo participo en el trabajo en equipo" el 65,2\% de los estudiantes manifestaron estar de acuerdo o totalmente de acuerdo, siendo un resultado coherente con el énfasis del desarrollo de esta competencia en las diferentes sesiones del Módulo.

En la competencia de adaptación, ante la afirmación "Me permitió mejorar mi capacidad para adaptarme a las nuevas situaciones y exigencias de los cursos" el $60 \%$ de los estudiantes manifestaron estar de acuerdo o muy de acuerdo. Esta competencia es el segundo valor agregado estratégico del Módulo (después de la socialización) en tanto permite a los estudiantes el desarrollo de estructuras subjetivas para afrontar los cambios académicos y sociales de la transición a la vida universitaria.

En síntesis, al analizar las premisas generales y específicas de vinculación del Módulo Metacognitivo con respecto a los cursos del segundo cuatrimestre 2018 (cuatrimestre posterior a la implementación del Módulo), se obtiene una percepción positiva superior al $60 \%$ en las diferentes habilidades desarrolladas y su importancia en los procesos de aprendizaje.

\section{Conclusiones}

La primera implementación del Ciclo Introductorio en el año 2018 consolidó el proceso de reforma curricular más profundo en la educación superior pública costarricense durante el siglo XXI. La aplicación de una política universitaria para el éxito académico en la transición (primer año de las carreras) mediante una profunda transformación de los planes de estudio con la inclusión de módulos con una innovadora apuesta epistémica, metodológica y evaluativa; marcaron un punto de inflexión en la vida universitaria de la UTN.

El Ciclo Introductorio encuentra en el Módulo Metacognitivo un referente fundamental por integrar las habilidades blandas de Pensar (indagación, pensamiento crítico, argumentación científica y resolución de problemas), Maneras de Trabajar (trabajo en equipo) y Herramientas para Trabajar (alfabetización digital), implementado durante todo el cuatrimestre en 13 sesiones sustentadas en el Aprendizaje Basado en Proyectos, trabajo colaborativo, 
procesos de reflexión sobre el aprendizaje alcanzado, socialización constante y una evaluación cualitativa.

Lo expuesto en el presente artículo permite establecer la pertinencia y relevancia del Módulo Metacognitivo a partir de las percepciones del estudiantado, tanto en su primera implementación (2018) como al finalizar el segundo cuatrimestre de las carreras (seguimiento). Destaca, en primer lugar, el alto nivel de participación de los estudiantes en la respuesta a los instrumentos $(41,5 \% \mathrm{du}-$ rante la implementación y un $27,4 \%$ en el seguimiento).

Los datos obtenidos evidencian, con especial énfasis, la valoración positiva de la vivencia del aprendizaje a partir de la práctica, realimentación y reflexión por parte de 7 de cada 10 estudiantes; 8 de cada 10 destaca el trabajo colaborativo realizado y las innovaciones de las actividades de aprendizaje. Esto demuestra una contextualización coherente con la propuesta epistémica y metodológica y podría conllevar la constitución de un aprendizaje significativo cuya relevancia sería longitudinal al plan de estudio de las carreras.

Con respecto a la percepción del dominio de las habilidades blandas, destaca la la diferencia establecida entre el dominio asumido como existen antes del Módulo y la percepción de dominio al finalizar el cuatrimestre. De esta forma, se establece una mejora en el dominio alto y muy alto de dos a tres veces superior al finalizar el Módulo (un promedio de $60 \%$ al finalizar el Módulo); así como una disminución de las percepciones de dominio muy bajo, bajo y medio.

Las habilidades tecnológicas vinculadas con Uso de Buscadores y Bases de Datos son las que presentan una mejora menor al promedio general, motivo por el cual se requiere un reforzamiento mediante uso de otras técnicas de mediación e inclusión de una plataforma de acompañamiento virtual.

Con respecto a la percepción de la influencia del Módulo Metacognitivo con los cursos del segundo cuatrimestre, es clara una influencia media-alta con un promedio superior al $60 \%$ en habilidades para presentación en público, investigación, uso de buscadores especializados, argumentación y debate, trabajo en equipo, identificar problemas y establecer rutas para crear soluciones y adaptación a nuevas situaciones. El alto nivel de valoración de la influencia tiene una posible correlación con el de estudiantes que manifestaron un dominio alto y muy alto en las diferentes habilidades blandas al finalizar la implementación del Módulo.

A partir de los hallazgos encontrados, surgen líneas de investigación cualitativa sobre las habilidades desarrolladas, la implementación de una evaluación cualitativa y sus repercusiones en las rupturas epistémicas de la población estudiantil, el desarrollo de las actividades didácticas en relación con las áreas de socialización, empoderamiento, identidad individual y colectiva, constitución de vínculos y la correlación con el éxito académico y las herramientas para favorecer la transición a la vida universitaria. Además, resulta recomendables estudios curriculares sobre la pionera asociación entre un ente externo (la Fundación Omar Dengo) y la Universidad Técnica Nacional como un mecanismo de innovación, mejora y aseguramiento de la calidad de los diseños curriculares.

Con respecto a las posibles acciones para una mejora de los resultados y la vinculación del Módulo Metacognitivo con los cursos del segundo cuatrimestre es recomendable la capacitación de los profesores que imparten cursos durante el primer año de las carreras en la temática de metacognición y sus implicaciones para el aprendizaje universitario. Así mismo, es recomendable la profundización de los procesos de capacitación para el personal docente que impartirá el Módulo en futuras implementaciones.

Finalmente, debe explicitarse que los resultados obtenidos en la percepción de los estudiantes de la primera implementación del Módulo Metacognitivo evidencian altos niveles de aceptación y respaldo, ratificando la importancia del Módulo Metacognitivo (y del Ciclo Introductorio en general) para la Universidad Técnica Nacional y la educación superior pública en general, en tanto se convierte en una estrategia referente para los desafíos de la transición a la educación superior.

\section{Referencias}

Errazuriz, J. (2018). Hacia una educación más integral: soft skills y ética. Calidad en la Educación, 34(18), pp. 119-130.

Garza, D. (2018). Conjugación de las habilidades para aprender en el desarrollo de los jóvenes Universitarios. Educiencia, 3(1), pp. 43-53.

González, V., Ferreira, J. y Barranco, A. (2018). Desarrollo de habilidades blandas y el uso del Sistema de Gestión del Aprendizaje en la elaboración de pro- 
yectos prácticos en una asignatura introductoria de Ingeniería Telemática. Cuaderno de Pedagogía Universitaria, 29(15), pp. 44-53

Hernández, R., Fernández, C. y Baptista, P. (2010). Metodología de la investigación. ( $5^{\mathrm{a}}$ ed.). México: Mac Graw Hill.

López, M; Mota; C, y Alvarado, R; 2018.Aprendizaje basado en problemas y sus aplicaciones en ingeniería. En: Inventio. 31(13), pp. 47-55

Programa Estado de la Nación (2017). Sexto Informe del Estado de la Educación. CONARE: Costa Rica.

Proyecto Tuning. (2007). Reflexiones y perspectivas de la educación superior en América Latina (Informe final Proyecto Tuning-América Latina 20042007). Bilbao: Universidad de Deusto.
Rodríguez, S; Fita, E y Torrado, M (2004). El rendimiento académico en la transición secundaria-universidad. Revista de Educación, (334), pp. 391-414

Silva, M. (2011). El primer año universitario. Un tramo crítico para el éxito académico Perfiles Educativos, vol. XXXIII, número especial.

Toruño, C. (2020). El currículum en el contexto costarricense: propuesta de definiciones para su conceptualización. Revista Ensayos Pedagógicos, 15(1), 39-59. https://doi.org/10.15359/rep.15-1.2

Universidad Técnica Nacional. (2016). Modelo Educativo. S.E: Costa Rica.

Universidad Técnica Nacional. (2016). Acuerdo 8-132016 Creación Ciclo Introductorio. Sesión Ordinaria No. 13-2016 del 29 de junio de 2016. 